\title{
Experiência Inicial com o Uso de Stents Eluidores de Everolimus em Pacientes do Mundo Real: Seguimento Clínico de Um Ano
}

\author{
Daniel Chamié ${ }^{1}$, Fausto Feres' ${ }^{1}$, J. Ribamar Costa Jr. ${ }^{1}$, Alexandre Abizaid ${ }^{1}$, Ricardo A. Costa', \\ Dimytri Siqueira1', Rodolfo Staico1', Galo Maldonado1, Luiz Alberto Mattos', Marinella Centemero', \\ Áurea Chaves ${ }^{1}$, Luiz Fernando Tanajura' ${ }^{1}$ Amanda G. M. R. Sousa'1', J. Eduardo Sousa ${ }^{1}$
}

\section{RESUMO}

Introdução: Os stents eluidores de everolimus mostraramse seguros e eficazes em estudos randomizados com pacientes selecionados e de baixa complexidade clínica e angiográfica. No entanto, estudos que avaliam seu impacto em pacientes mais complexos da prática clínica corrente são escassos. Método: No total, foram avaliados, prospectivamente, 193 pacientes tratados apenas com stents eluidores de everolimus. Os únicos critérios de exclusão foram reestenose de outro stent farmacológico, intervenção em enxertos de veia safena, e infarto agudo do miocárdio com elevação do segmento ST. O desfecho primário foi a ocorrência de eventos cardíacos adversos maiores, definidos como a combinação de morte de origem cardíaca, infarto do miocárdio e revascularização do vaso-alvo. Resultados: A média das idades foi de 60,6 \pm 11,8 anos. Metade da população era do sexo feminino e $36,8 \%$ eram portadores de diabetes. Doença multiarterial estava presente em $61,6 \%$ dos pacientes. Foi tratada 1,8 $\pm 0,66$ lesão em $1,73 \pm 0,9$ vaso, com $2,27 \pm 0,9$ stents por paciente. A maioria das lesões $(70,6 \%)$ era do tipo B2/C, bifurcações verdadeiras foram tratadas em $37,3 \%$ dos casos e calcificação moderada a grave estava presente em $17,4 \%$ das lesões. Eventos cardíacos adversos maiores ocorreram em $6,7 \%$ dos pacientes ao final de um ano, principalmente à custa de eventos da fase hospitalar. Houve apenas um caso $(0,5 \%)$ de trombose subaguda provável do stent. Não houve casos de trombose tardia. Conclusões: A presente análise sugere que o uso de stents eluidores de everolimus é seguro e eficaz em pacientes complexos da prática clínica diária, com baixas taxas de eventos cardíacos adversos maiores até o final do primeiro ano. Seguimento mais longo e com maior número de

\section{ABSTRACT}

Early Experience with Everolimus-Eluting Stents in Real-world Patients: One-Year Clinical Follow-Up

Background: Everolimus-eluting stents have proved to be safe and effective in the treatment of low-risk, selected patients, from randomized trials. Nonetheless, studies assessing their impact on more complex real-world patients are rare. Method: We prospectively evaluated 193 patients treated with everolimus-eluting stents alone. The only exclusion criteria were: in-stent restenosis of another drug-eluting stent, percutaneous coronary intervention of a saphenous vein graft and acute myocardial infarction with ST segment elevation. The primary endpoint was the incidence of major adverse cardiac events (MACE), defined as the composite of cardiac death, myocardial infarction and target vessel revascularization. Results: Mean age was $60.6 \pm 11.8$ years. Half of the patients were women and $36.8 \%$ were diabetics. Multivessel disease was found in $61.6 \%$ of the patients. Patients had $1.8 \pm 0.66$ lesion treated in $1.73 \pm 0.9$ vessel, with a mean of $2.27 \pm 0.9$ stents per patient. The majority $(70.6 \%)$ of the lesions were type B2/C, true bifurcations were treated in $37.3 \%$ of the cases and moderate to severe calcifications were observed in $17.4 \%$ of the lesions. The 1 -year MACE rate was $6.7 \%$, mainly due to the occurrence of in-hospital events. There was only one $(0.5 \%)$ case of probable subacute stent thrombosis. No cases of late stent thrombosis were observed. Conclusions: The present analysis suggests that the use of everolimus eluting stents is safe and effective in the treatment of complex patients from the daily clinical practice, with low major adverse cardiac events rate in the 1-year follow-up. Longer follow-up with

\footnotetext{
1 Instituto Dante Pazzanese de Cardiologia - São Paulo, SP, Brasil. Correspondência: Fausto Feres. Av. Dr. Dante Pazzanese, 500 Ibirapuera - São Paulo, SP, Brasil - CEP 04012-180 E-mail: faustoferes@hotmail.com

Recebido em: 2/10/2009 • Aceito em: 4/12/2009
} 
Chamié D, et al. Experiência Inicial com o Uso de Stents Eluidores de Everolimus em Pacientes do Mundo Real: Seguimento Clínico de Um Ano. Rev Bras Cardiol Invas. 2009;17(4):484-90.

pacientes é necessário para confirmação desses resultados promissores.

DESCRITORES: Doença das coronárias. Reestenose coronária/terapia. Stents farmacológicos. Paclitaxel/administração \& dosagem. Sirolimus/administração \& dosagem. Trombose/prevenção \& controle. Seguimentos. Resultado de tratamento. ndubitavelmente, o desenvolvimento e a aplicação clínica dos stents farmacológicos revolucionaram a prática da cardiologia intervencionista. Comparados aos stents não-farmacológicos, os stents farmacológicos de "primeira geração", liberadores de sirolimus e paclitaxel, mostraram-se altamente eficazes em reduzir a formação de hiperplasia neointimal e, consequentemente, as taxas de reestenose angiográfica e a necessidade de novas revascularizações da lesão-alvo ${ }^{1-3}$. Entretanto, a observação de maiores taxas de trombose tardia e muito tardia dos stents eluidores de sirolimus e de paclita$\mathrm{xel}^{4-7}$, bem como o maior grau de endotelização incompleta $^{8}$ desses dispositivos, quando comparados aos stents não-farmacológicos, tornaram-se motivos de recente preocupação com essa nova tecnologia.

Com o objetivo de melhorar o perfil de segurança dos stents farmacológicos, sem perda de sua efetividade, foi desenvolvido um stent eluidor de everolimus (Abbott Vascular, Santa Clara, Estados Unidos), que libera o agente antiproliferativo a partir de um polímero biocompatível, de fina espessura $(7,8 \mu \mathrm{m})$, durável e não-adesivo, aplicado sobre a superfície de um stent de cromocobalto, de hastes finas $(0,0813 \mathrm{~mm})$, altamente flexível.

Os ensaios clínicos randomizados SPIRIT II ${ }^{9}$ e III $^{10}$ demonstraram a superioridade tanto angiográfica como clínica desse novo dispositivo comparativamente ao stent eluidor de paclitaxel em pacientes de baixa complexidade. No SPIRIT IV ${ }^{11}$, com maior número de pacientes, a superioridade clínica dos stents eluidores de everolimus foi confirmada em pacientes de moderada complexidade. Informações acerca de seu uso em populações mais complexas, representativas da prática clínica diária, são escassas e limitadas ${ }^{12}$. Nesse sentido, apresentamos a experiência inicial de nosso grupo com o uso dos stents eluidores de everolimus em pacientes de maior complexidade.

\section{MÉTODO}

\section{Desenho do estudo e seleção dos pacientes}

Este estudo consiste de um registro prospectivo, unicêntrico e de braço único, que visa a avaliar a segurança e a efetividade do uso dos stents eluidores de everolimus em pacientes da prática clínica corrente.

Entre dezembro de 2007 e dezembro de 2009, 243 pacientes com lesões coronárias foram tratados a larger number of patients is required to confirm these promising results.

DESCRIPTORS: Coronary disease. Coronary restenosis/therapy. Drug-eluting stents. Paclitaxel/administration \& dosage. Sirolimus/administration \& dosage. Thrombosis/prevention $\&$ control. Follow-up studies. Treatment outcome.

com o uso de stents eluidores de everolimus. Até o presente momento, 193 pacientes $(79,4 \%)$ completaram um ano de seguimento, compreendendo, portanto, a população analisada neste manuscrito. Os pacientes foram pouco selecionados e os critérios de exclusão foram reestenose de outro stent farmacológico, intervenção em enxertos de veia safena, e intervenção coronária percutânea primária em pacientes com infarto agudo do miocárdio com elevação do segmento ST.

Declaração de consentimento informado foi obtida de todos os pacientes, e o protocolo do registro foi aprovado pelo Comitê de Ética de nossa Instituição.

\section{Procedimento de intervenção coronária}

O protocolo antiplaquetário envolveu a utilização do ácido acetilsalicílico e do clopidogrel. O pré-tratamento (ácido acetilsalicílico 500 mg e clopidogrel $300 \mathrm{mg}$ ) foi realizado 24 horas antes do procedimento. Após a intervenção, o ácido acetilsalicílico foi mantido indefinidamente, na dose de $100 \mathrm{mg} / \mathrm{dia}$, e o clopidogrel, na dose de $75 \mathrm{mg}$, por 12 meses.

Todos os procedimentos foram realizados de acordo com as diretrizes vigentes ${ }^{13}$. Durante o procedimento, heparina não-fracionada foi administrada para todos os pacientes na dose de $100 \mathrm{UI} / \mathrm{kg}$, com o objetivo de manter o tempo de coagulação ativada entre 250 e 350 segundos. O uso de inibidores da glicoproteína Ilb/Illa ficou a critério do operador. Pré-dilatação não era obrigatória e a pós-dilatação dos stents era recomendada em caso de estenose residual > 30\% por estimativa visual.

\section{Objetivos e definições}

O objetivo primário deste registro foi avaliar a incidência de eventos cardíacos adversos maiores, definidos como a ocorrência combinada de morte de origem cardíaca, infarto não-fatal ou revascularização do vaso-alvo. Os objetivos secundários compreenderam a ocorrência isolada de morte de origem cardíaca, infarto não-fatal, revascularização do vaso-alvo, revascularização da lesão-alvo, trombose definitiva e/ou provável dos stents e a combinação de morte de origem cardíaca e infarto não-fatal.

Todos os óbitos foram considerados de origem cardíaca, a menos que uma causa não-cardíaca fosse 
Chamié D, et al. Experiência Inicial com o Uso de Stents Eluidores de Everolimus em Pacientes do Mundo Real: Seguimento Clínico de Um Ano. Rev Bras Cardiol Invas. 2009;17(4):484-90.

inequivocamente identificada. Infarto não-fatal incluiu: infarto periprocedimento, definido em conformidade com as recomendações de uma força tarefa global (ESC/ACC/AHA/WHF) ${ }^{14}$, como elevação nos níveis de troponina ou CK-MB maior que três vezes o limite superior da normalidade até 48 horas após a intervenção coronária percutânea; e infarto espontâneo, definido conforme recomendação do Academic Research Consortium $(\mathrm{ARC})^{15}$ como qualquer elevação de troponina ou CK-MB acima do valor superior de normalidade do laboratório, não relacionada com procedimentos de intervenção coronária percutânea, em qualquer momento após a intervenção índex. Os eventos foram ainda divididos em infartos com onda $Q$ ou sem onda $Q$, de acordo com a presença ou ausência de ondas Q patológicas (ondas Q > 0,04 segundo em duas derivações contíguas) no eletrocardiograma. Revascularização do vaso-alvo foi definida como novo procedimento de intervenção percutânea ou cirurgia de revascularização do miocárdio de uma lesão no mesmo vaso epicárdico tratado no procedimento índex. Revascularização da lesão-alvo foi definida como novo procedimento de intervenção percutânea da lesãoalvo ou cirurgia de revascularização do miocárdio do vaso-alvo realizados em consequência de reestenose no segmento da lesão-alvo. Lesão-alvo foi definida como o segmento intrastent acrescido de $5 \mathrm{~mm}$ proximais e distais a seus bordos. Reestenose foi definida como porcentual de estenose $>50 \%$ no segmento da lesão-alvo. Para realização de revascularização do vasoalvo ou revascularização da lesão-alvo era necessária a presença de sintomas ou evidência funcional de isquemia, bem como a documentação de lesão com $>50 \%$ de porcentual de estenose, no vaso-alvo ou na lesão-alvo, respectivamente. Trombose dos stents também foi definida conforme as recomendações do $\mathrm{ARC}^{15}$, sendo classificada de acordo com o nível de evidência (definitiva, provável ou possível) e com o tempo de ocorrência (aguda, até 24 horas; subaguda, entre 24 horas e 30 dias; e tardia, entre 30 dias e um ano). Como o presente estudo avalia a ocorrência dos desfechos clínicos até um ano, a taxa de trombose muito tardia (maior que um ano) não será apresentada. Sucesso angiográfico foi definido como obtenção de lesão residual menor que $30 \%$ e fluxo coronário TIMI 3. Nos casos de lesões em bifurcações, em que o tratamento do ramo lateral foi apenas realizado com cateter-balão, o critério de sucesso angiográfico foi definido como presença de lesão residual menor que 50\%, com fluxo coronário TIMI 3 e ausência de dissecções.

\section{Análise estatística}

Na análise estatística descritiva, as variáveis categóricas foram expressas como frequências absolutas e porcentuais. As variáveis contínuas foram expressas como média \pm desvio padrão. Como o presente manuscrito visa apenas a apresentar os resultados de um registro prospectivo, de braço único, não foram realizadas comparações. Curva com a incidên- cia cumulativa do desfecho primário foi construída pelo método de Kaplan-Meier, para demonstração da ocorrência dos eventos em função do tempo. Para esta análise, foi utilizado o programa SPSS 13.0 for Windows.

\section{RESULTADOS}

As características clínicas e angiográficas de base, bem como dados referentes ao procedimento, são apresentadas, respectivamente, nas Tabelas 1, 2 e 3 .

A média das idades da população analisada foi de 60,6 anos. Metade dos pacientes era do sexo feminino e $36,8 \%$ eram portadores de diabetes melito. A indicação clínica mais prevalente para a realização da intervenção coronária percutânea foi angina estável (43,5\% dos casos). Entre os pacientes avaliados, $30 \%$ da população era portadora de síndrome coronária aguda, dos quais $77,9 \%$ tinham critérios de risco moderado a alto pela classificação $\mathrm{TIMI}^{16}$.

A maioria dos pacientes $(61,6 \%)$ era portadora de doença multiarterial, tendo sido tratado 1,73 $\pm 0,9$ vaso por paciente. O vaso-alvo mais frequentemente tratado foi a artéria descendente anterior (58,1\%). Foi tratada 1,8 \pm 0,66 lesão por paciente, sendo a maioria $(70,6 \%)$ do tipo B2/C. Bifurcações verdadeiras representaram $37,3 \%$ das lesões tratadas. Calcificação moderada a grave esteve presente em $17,4 \%$ das lesões.

TABELA 1

Características clínicas basais

\begin{tabular}{lc}
\hline & $\mathbf{n}=\mathbf{1 9 3}$ \\
\hline Idade, média $\pm \mathrm{DP}$ & $60,6 \pm 11,8$ \\
Sexo feminino, $\mathrm{n}(\%)$ & $98(50,7)$ \\
Hipertensão arterial, $\mathrm{n}(\%)$ & $169(87,6)$ \\
Diabetes melito, $\mathrm{n}(\%)$ & $71(36,8)$ \\
$\quad$ Dependente de insulina & $32(16,6)$ \\
Dislipidemia, $\mathrm{n}(\%)$ & $144(74,6)$ \\
Tabagismo atual, $\mathrm{n}(\%)$ & $31(16,1)$ \\
Insuficiência renal crônica, $\mathrm{n}(\%)$ & $49(25,4)$ \\
Infarto prévio, $\mathrm{n}(\%)$ & $93(48,2)$ \\
ICP prévia, $\mathrm{n}(\%)$ & $26(13,5)$ \\
CRVM prévia, $\mathrm{n}(\%)$ & $11(5,7)$ \\
Indicação, $\mathrm{n}(\%)$ & \\
Isquemia silenciosa & $50(25,9)$ \\
$\quad$ Angina estável & $84(43,5)$ \\
SCA SSST & $59(30,6)$ \\
Risco TIMI moderado/alto & $46(77,9)$ \\
\hline CRVM = cirurgia de revascularização do miocárdio; DP = \\
desvio padrão; ICP = intervenção coronária percutânea; $\mathrm{n}=$ \\
número de pacientes; SCA SSST = síndrome coronária aguda \\
sem supradesnivelamento do segmento ST. \\
\hline
\end{tabular}


Chamié D, et al. Experiência Inicial com o Uso de Stents Eluidores de Everolimus em Pacientes do Mundo Real: Seguimento Clínico de Um Ano. Rev Bras Cardiol Invas. 2009;17(4):484-90.

TABELA 2

Características angiográficas basais

\begin{tabular}{|c|c|}
\hline & $\mathrm{n}=193$ \\
\hline \multicolumn{2}{|l|}{ Pacientes com lesão > 50\%, n (\%) } \\
\hline 1 vaso & $74(38,4)$ \\
\hline 2 vasos & $97(50,2)$ \\
\hline 3 vasos & $22(11,4)$ \\
\hline \multicolumn{2}{|l|}{$\begin{array}{l}\text { Vasos com lesão }>50 \% \text { por paciente, } \\
\text { média } \pm \text { DP }\end{array}$} \\
\hline \multicolumn{2}{|l|}{ Vaso-alvo, n (\%) } \\
\hline Descendente anterior & $194 / 334(58,1)$ \\
\hline Circunflexa & $92 / 334(27,5)$ \\
\hline Coronária direita & $43 / 334(12,9)$ \\
\hline Ramo intermédio & $5 / 334(1,5)$ \\
\hline Lesões por paciente, média \pm DP & $1,8 \pm 0,66$ \\
\hline Lesões tipo B2/C*, n (\%) & $236 / 334(70,6)$ \\
\hline Calcificação moderada a grave, n (\%) & $58(17,4)$ \\
\hline Bifurcações, n (\%) & $72(37,3)$ \\
\hline Reestenose de SNF, n (\%) & $8(4,2)$ \\
\hline Oclusões crônicas, n (\%) & $3(1,5)$ \\
\hline DRV, mm (média \pm DP) & $2,44 \pm 1,01$ \\
\hline Extensão da lesão, mm (média \pm DP) & $14,11 \pm 8,79$ \\
\hline DLM, mm (média \pm DP) & $0,94 \pm 0,48$ \\
\hline DS, \% (média × DP) & $55,59 \pm 23,26$ \\
\hline \multicolumn{2}{|c|}{$\begin{array}{l}\text { * Classificação do American College of Cardiology/American } \\
\text { Heart Association. } \\
\text { DLM = diâmetro luminal mínimo; DP = desvio padrão; DRV = } \\
\text { diâmetro de referência do vaso; DS = diâmetro de estenose; } \\
\mathrm{n}=\text { número de pacientes; SNF = stent não-farmacológico. }\end{array}$} \\
\hline
\end{tabular}

O acesso vascular para realização dos procedimentos foi predominantemente a via femoral $(85,5 \%)$. Foi utilizada média de 2,27 stents por paciente. Revascularização completa foi obtida em $83,9 \%$ dos pacientes. O ultrassom intracoronário como ferramenta para guiar o implante dos stents foi utilizado em 23,3\% dos casos. Pós-dilatação dos dispositivos implantados foi utilizada em $89,6 \%$ dos casos, e sucesso angiográfico foi obtido na maioria $(97,9 \%)$ das lesões tratadas.

\section{Seguimento clínico até um ano}

Dados acerca do seguimento clínico até um ano após o procedimento índex foram obtidos de todos os pacientes, por consulta ambulatorial ou contato telefônico. A ocorrência de eventos cardíacos adversos maiores no final de um ano foi de $6,73 \%$. A Figura 1 ilustra graficamente a incidência cumulativa dos eventos, enquanto a Tabela 4 apresenta a taxa de ocorrência dos desfechos nos períodos intra-hospitalar e entre a alta hospitalar e 12 meses de seguimento. Houve dois casos de óbito de origem cardíaca na fase intrahospitalar. Outro paciente apresentou morte súbita oito
TABELA 3

Dados do procedimento

\begin{tabular}{|c|c|}
\hline & $n=193$ \\
\hline \multicolumn{2}{|l|}{ Acesso vascular, n (\%) } \\
\hline Femoral & $165(85,5)$ \\
\hline Radial & $28(14,5)$ \\
\hline Cateter-guia calibre 7 F, n (\%) & $71(36,8)$ \\
\hline Revascularização completa, n (\%) & $162(83,9)$ \\
\hline Uso de inibidores da GP IIb/IIla, n (\%) & $11(5,7)$ \\
\hline Uso de USIC, n (\%) & $45(23,3)$ \\
\hline Stents/paciente, média \pm DP & $2,27 \pm 0,9$ \\
\hline Pré-dilatação, n (\%) & $133(68,7)$ \\
\hline Pós-dilatação, n (\%) & $173(89,6)$ \\
\hline $\begin{array}{l}\text { Pressão de dilatação máxima, } \\
\text { atm (média } \pm \text { DP) }\end{array}$ & $17,49 \pm 3,52$ \\
\hline $\begin{array}{l}\text { Diâmetro dos stents, } \\
\text { mm (média } \pm \text { DP) }\end{array}$ & $2,88 \pm 0,35$ \\
\hline $\begin{array}{l}\text { Comprimento dos stents, } \\
\text { mm (média } \pm \text { DP) }\end{array}$ & $19,01 \pm 5,49$ \\
\hline Volume de contraste, $\mathrm{ml}$ (média \pm DP) & $162,4 \pm 80,5$ \\
\hline Sucesso angiográfico, n (\%) & $189(97,9)$ \\
\hline
\end{tabular}

$\mathrm{DP}$ = desvio padrão; GP Ilb/IIla = glicoproteína Ilb/IIla; $\mathrm{n}=$ número de pacientes; USIC = ultrassom intracoronário.

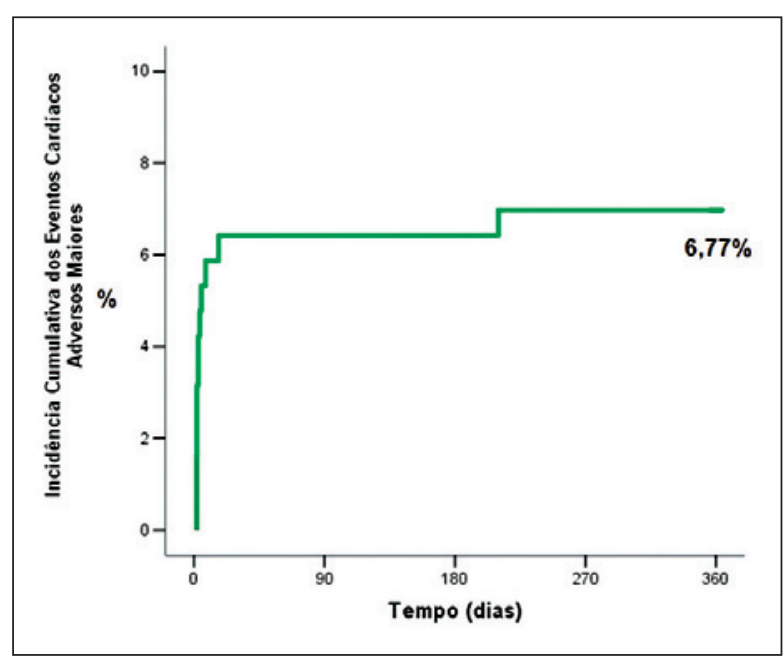

Figura 1 - Incidência cumulativa dos eventos cardíacos adversos maiores até o seguimento de um ano.

dias após o procedimento índex. Esse paciente, por decisão própria, havia descontinuado o clopidogrel e seu óbito foi considerado consequente a provável trombose de stent (subaguda). No período intra-hospitalar, 8 pacientes apresentaram elevação de CK-MB maior que três vezes o limite superior da normalidade, caracterizando infartos periprocedimento. Outro paciente foi readmitido após 17 dias do procedimento índex, 
TABELA 4 Desfechos clínicos

\begin{tabular}{lc}
\hline & $\mathbf{n}=\mathbf{1 9 3}$ \\
\hline Período intra-hospitalar & $10(6,2)$ \\
ECAM, n (\%) & $2(1)$ \\
Óbito cardíaco, n (\%) & $8(4,1)$ \\
Infarto do miocárdio, n (\%) & 0 \\
RLA, n (\%) & $3(1,6)$ \\
Período entre a alta hospitalar até um ano & $1(0,5)$ \\
ECAM, n (\%) & $1(0,5)$ \\
Óbito cardíaco, n (\%) & $1(0,5)$ \\
Infarto do miocárdio, n (\%) & 0 \\
RVA, n (\%) & $13(6,7)$ \\
RLA, n (\%) & 0 \\
Total ECAM, n (\%) & $1(0,5)$ \\
Trombose definitiva/provável de stents, $\mathrm{n}(\%)$ & 0 \\
$\quad$ Aguda & \\
Subaguda & \\
$\quad$ Tardia & \\
\hline ECAM = eventos cardíacos adversos maiores; $\mathrm{n}=$ número \\
de pacientes; RLA = revascularização da lesão-alvo; RVA = \\
revascularização do vaso-alvo.
\end{tabular}

com quadro de dor torácica anginosa e elevação dos marcadores de necrose miocárdica, sem novas alterações eletrocardiográficas. No momento da reinternação, foi submetido a nova cinecoronariografia diagnóstica, que revelou patência dos stents implantados com manutenção do resultado agudo, sem novas lesões em outros territórios. Esse evento foi considerado, portanto, como novo infarto, mas não como trombose dos stents. Não houve casos de revascularização da lesãoalvo até o seguimento de um ano. Cabe mencionar, no entanto, que nesse período de seguimento ocorreram dois casos de reestenose de stent em ramos laterais de lesões em bifurcação, tratados clinicamente. Um paciente voltou a apresentar quadro de angina estável sete meses após o procedimento índex, com nova cinecoronariografia revelando progressão de doença no segmento proximal do vaso-alvo, proximalmente a um stent eluidor de everolimus implantado e que se apresentava patente e sem reestenose.

\section{DISCUSSÃO}

Neste manuscrito é reportada a experiência inicial do nosso centro com o uso de stents eluidores de everolimus, apresentando as taxas de ocorrência de eventos cardíacos adversos até um ano de pacientes minimamente selecionados, representativos da prática clínica diária.

A segurança e a efetividade dos stents eluidores de everolimus em pacientes selecionados e de baixa complexidade já foram demonstradas previamente ${ }^{9,10}$. No estudo SPIRIT II, conduzido na Europa, Nova Zelândia e Índia, 300 pacientes foram randomizados de forma 3:1 para receber um stent eluidor de everolimus $(n=223)$ ou stents eluidores de paclitaxel $(n=77)$. Os stents eluidores de everolimus demonstraram ser não apenas não-inferiores como também superiores aos stents eluidores de paclitaxel, com menor perda luminal tardia aos seis meses $(0,11 \pm 0,27 \mathrm{~mm}$ vs. 0,36 \pm $0,39 \mathrm{~mm} ; \mathrm{P}<0,0001)$. No entanto, provavelmente em função do pequeno número da amostra, nenhuma diferença foi encontrada na ocorrência de eventos cardíacos adversos.

No estudo SPIRIT III, conduzido nos Estados Unidos, 1.002 pacientes, também de baixa complexidade, foram randomizados de forma 2:1 para receber um stent eluidor de everolimus $(n=669)$ ou stent eluidor de paclitaxel $(\mathrm{n}=333)$. O desfecho primário desse ensaio foi a perda luminal tardia aos oito meses. Novamente, os stents eluidores de everolimus demonstraram eficácia superior, com redução significativa da perda luminal tardia $(0,14 \pm 0,41 \mathrm{~mm}$ vs. $0,28 \pm 0,48 \mathrm{~mm}$; $P \leq 0,004)$. Nesse estudo, os stents eluidores de everolimus demonstraram ser não só não-inferiores aos stents eluidores de paclitaxel em relação à falência do vaso-alvo aos nove meses $(7,2 \%$ vs. $9 \%$; $\mathrm{P}<0,001)$, como também em relação à redução dos eventos cardíacos adversos combinados aos nove meses $(4,6 \%$ vs. $8,1 \% ; P=0,03)$ e um ano $(6 \%$ vs. $10,3 \% ; P=0,02)$.

O recém-apresentado estudo SPIRIT IV ${ }^{11}$, conduzido em 66 centros norte-americanos, é o maior ensaio randomizado avaliando o desempenho de stents farmacológicos já realizado até o momento, com poder estatístico adequado para avaliar a ocorrência de desfechos clínicos. Nesse estudo, 3.687 pacientes foram randomizados de forma 2:1 para receber stents eluidores de everolimus ( $n=2.458$ ) ou stents eluidores de paclitaxel $(n=1.229)$. A inclusão foi ligeiramente mais liberal que nos estudos anteriores, permitindo a presença de até três lesões de novo em coronárias nativas (máximo de duas lesões por vaso epicárdico). Nesse estudo, o uso de stents eluidores de everolimus resultou em taxas significativamente menores de falência do vaso-alvo aos 12 meses ( $3,9 \%$ vs. $6,6 \%$; $P=0,0008)$, de revascularização da lesão-alvo $[2,5 \%$ vs. $4,6 \%$; $P$ (não-inferioridade $)<0,0001$ e $P$ (superioridade $)=0,001$, e de desfecho combinado de morte e infarto relacionado ao vaso-alvo $[2,2 \%$ vs. $3,2 \%$; P (não-inferioridade) $<0,0001$ e $P$ (superioridade) $=0,09]$. Além disso, as taxas de trombose definitiva e provável também foram significativamente menores com os stents eluidores de everolimus $(0,29 \%$ vs. $1,06 \% ; P=0,003)$.

O tratamento de pacientes de maior complexidade com stents eluidores de everolimus foi reportada recentemente no registro Xience $V$ Stent Evaluated at Rotterdam Cardiac Hospital (X-SEARCH) ${ }^{12}$, conduzido em Roterdã (Holanda). Naquela instituição, os stents 
Chamié D, et al. Experiência Inicial com o Uso de Stents Eluidores de Everolimus em Pacientes do Mundo Real: Seguimento Clínico de Um Ano. Rev Bras Cardiol Invas. 2009;17(4):484-90.

eluidores de everolimus passaram a ser o dispositivo padrão para intervenção coronária percutânea após tornarem-se disponíveis comercialmente na Europa em março de 2007. Os autores reportam os resultados de seis meses de 649 pacientes consecutivos, tratados entre março e outubro de 2007. Nesse registro, não foi imputada qualquer restrição clínica ou anatômica ao uso dos stents eluidores de everolimus. Ao final de seis meses, a ocorrência de eventos cardíacos adversos maiores foi de 9,2\%. Em comparação com o registro X-SEARCH, nosso registro, apesar de ter incluído pacientes mais jovens (60 anos vs. 64 anos), tinha maior prevalência de mulheres $(50,7 \%$ vs. $28,4 \%)$, diabéticos (36,8\% vs. 20,8\%) e história de infarto prévio $(48,2 \%$ vs. $25,9 \%)$. Quanto à apresentação clínica, $39,3 \%$ dos pacientes do registro X-SEARCH tinham infarto do miocárdio com elevação do segmento ST, com $6 \%$ em choque cardiogênico. A prevalência de angina instável ou infarto do miocárdio sem elevação do segmento ST foi de $20,2 \%$, enquanto em nosso registro foi de $30,6 \%$. Doença multiarterial foi mais prevalente na nossa população $(61,6 \%$ vs. $50,2 \%)$ e a descendente anterior foi a artéria mais frequentemente tratada nos dois grupos. Em ambos os estudos foi tratada, em média, 1,8 lesão por paciente, com utilização de 2,1 stents por paciente no X-SEARCH e 2,3 stents por paciente no nosso registro. A prevalência de bifurcações verdadeiras foi maior no nosso estudo $(37,3 \%$ vs. $22,2 \%)$.

Apesar da alta complexidade clínica e angiográfica, a incidência de eventos cardíacos adversos maiores na nossa população foi notavelmente baixa $(6,73 \%)$, com a maioria dos eventos ocorrendo na fase intra-hospita$\operatorname{lar}(6,21 \%)$. Chama a atenção a ausência de revascularizações da lesão-alvo até o seguimento de um ano nessa população, confirmando a eficicácia antiproliferativa desse dispositivo. Acreditamos, também, que a técnica de implante desempenhou papel importante nesse aspecto. A realização de pós-dilatações com balões nãocomplacentes, de comprimento menor que o stent implantado e insuflados com altas pressões foi bastante utilizada em nossa coorte, com cifras bem acima daquelas reportadas habitualmente na literatura. Além disso, o uso frequente do ultrassom intracoronário, auxiliando na identificação dos segmentos de referência "normais" do vaso, na escolha adequada dos stents e permitindo a cobertura completa das lesões, também pode ter influenciado os resultados.

Já foi claramente demonstrado que os stents farmacológicos de primeira geração estão associados a maior taxa de trombose, quando comparados aos stents não-farmacológicos. No presente registro, com pacientes de alta complexidade, a ocorrência de trombose dos stents foi extremamente rara $(0,51 \%)$, não sendo constatado qualquer caso de trombose tardia. O registro X-SEARCH corrobora esses números, com taxa total de trombose dos stents eluidores de everolimus de $0,6 \%$ nos primeiros seis meses, nenhuma delas tardia.

\section{LIMITAÇÕES}

A principal limitação do presente estudo é o tamanho da população incluída, que pode ser insuficiente para avaliar desfechos de segurança. No entanto, seu valor reside no fato de representar uma das primeiras experiências clínicas com o stent eluidor de everolimus, em pacientes de alta complexidade clínica e angiográfica, mais representativos da prática clínica diária.

Deve ser mencionado também o fato de ser um registro de braço único, observacional e não-randomizado.

\section{CONCLUSÕES}

$\mathrm{Na}$ presente análise envolvendo pacientes complexos da prática diária, o novo stent farmacológico eluidor de everolimus mostrou-se eficaz, com baixa taxa de eventos cardíacos adversos até um ano de seguimento clínico. Adicionalmente, observamos taxas de trombose de stent menores que $1 \%$, inferiores ao que tem sido reportado com os stents farmacológicos de primeira geração em cenários de semelhante complexidade.

\section{CONFLITO DE INTERESSES}

Os autores declararam inexistência de conflito de interesses.

\section{REFERÊNCIAS BIBLIOGRÁFICAS}

1. Morice MC, Serruys PW, Sousa JE, Fajadet J, Ban Hayashi E, Perin M, Colombo A, Schuler G, Barragan P, Guagliumi G, Molnar F, Falotico R; RAVEL Study Group. Randomized Study with the Sirolimus-Coated Bx Velocity Balloon-Expandable Stent in the Treatment of Patients with de Novo Native Coronary Artery Lesions. A randomized comparison of a sirolimus-eluting stent with a standard stent for coronary revascularization. N Engl J Med. 2002;346(23):1773-80. Comment in: Surg Neurol. 2003;59(3):146-7. N Engl J Med. 2002;347(16):1285; author reply 1285. N Engl J Med. 2002; 346(23):1770-1.

2. Sousa JE, Costa M, Abizaid A, Feres F, Seixas AC, Tanajura $L F$, et al. Four-year angiographic and intravascular ultrasound follow-up of patients treated with sirolimus-eluting stents. Circulation. 2005;111(18):2326-9.

3. Stone GW, Ellis SG, Cox DA, Hermiller J, O'Shaughnessy C, Mann JT, Turco M, Caputo R, Bergin P, Greenberg J, Popma J, Russell ME; TAXUS-IV-Investigators. A polymer-based, paclitaxel-eluting stent in patients with coronary artery disease. TAXUS-IV Investigators. N Engl J Med. 2004;350(3):221-31. Comment in: N Engl J Med. 2004;350(20):2099-100; author reply 2099-100. N Engl J Med. 2005;353(22):2404-8; author reply 2404-8. N Engl J Med. 2004;350(3):211-2.

4. Camenzind E, Steg PG, Wijns W. Stent thrombosis late after implantation of first-generation drug-eluting stents: a cause for concern. Circulation. 2007;115(11):1440-55; discussion 1455. Comment in: Circulation. 2007;116(16):e389; author reply e390. Circulation. 2007;116(16):e388; author reply e390. Comment on: Circulation. 2007;115(11):1433-9 discussion 1439.

5. Daemen J, Wenawesser P, Tsuchida K, Abrecht L, Vaina S, 
Morger C, et al. Early and late coronary stent thrombosis of sirolimus-eluting and paclitaxel-eluting stents in routine clinical practice: data from a large two-institutional cohort study. Lancet. 2007;369(9562):667-78. Comment in: Eurolntervention. 2008;4 Suppl C:C52-4. Lancet. 2007;369(9575):1785; author reply 1785-6. Lancet. 2007;369(9562):619-21.

6. Lagerqvist B, James SK, Stenestrand U, Lindbäck J, Nilsson T, Wallentin L; SCAAR Study Group. Long-term outcomes with drug-eluting stents versus bare-metal stents in Sweden. N Engl J Med. 2007;356(10):1009-19. Comment in: Eurolntervention. 2008;4 Suppl C:C49-51. N Engl J Med. 2007; 356(10):1059-60. N Engl J Med. 2007;356(10):981-4.

7. Stone GW, Moses JW, Ellis SG, Schofer J, Dawkins KD, Morice MC, et al. Safety and efficacy of sirolimus- and paclitaxel-eluting coronary stents. N Engl J Med. 2007; 356(10):998-1008. Comment in: N Engl J Med. 2007;356(10): 1059-60.

8. Finn AV, Joner M, Nakazawa G, Kolodgie F, Newell J, John $M C$, et al. Pathological correlates of late drug-eluting stent thrombosis: strut coverage as a marker of endothelialization. Circulation. 2007;115(18):2435-41. Comment in: Circulation. 2007;116(22):e549; author reply e550.

9. Serruys PW, Ruygrok P, Neuzner J, Piek JJ, Seth A, Schofer J, et al. A randomized comparison of an everolimus-eluting coronary stent with a paclitaxel-eluting coronary stent: the SPIRIT II trial. Eurolntervention. 2006;2(3):286-94.

10. Stone GW, Midei M, Newman W, Sanz M, Hermiller JB, Williams J, Farhat N, Mahaffey KW, Cutlip DE, Fitzgerald PJ, Sood P, Su X, Lansky AJ; SPIRIT III Investigators. Comparison of an everolimus-eluting stent and a paclitaxel-eluting stent in patients with coronary artery disease: a randomized trial. JAMA. 2008;299(16):1903-13. Comment in: JAMA. 2008; 299(16):1952-3.
11. Stone GW, for the SPIRIT IV Investigators. A prospective, randomized trial comparing an everolimus-eluting stent and a paclitaxel-eluting stent in patients with coronary artery disease. One year clinical results. In: 21st Annual Scientific Symposium Transcatheter Cardiovascular Therapeutics (TCT 2009). 2009 Sep 21-26 São Francisco (CA), EUA.

12. Onuma Y, Kukreja N, Piazza N, Eindhoven J, Girasis C, Schenkeveld $\mathrm{L}$, van Domburg R, Serruys PW; Interventional Cardiologists of the Thoraxcenter (2000 to 2007). The everolimus-eluting stent in real-world patients: 6-month follow-up of the X-SEARCH (Xience V Stent Evaluated at Rotterdam Cardiac Hospital) registry. J Am Coll Cardiol. 2009;54(3):269-76.

13. King SB 3rd, Smith SC Jr, Hirshfeld JW Jr, Jacobs AK, Morrison DA, Williams DO, et al. 2007 focused update of the ACC/ $\mathrm{AHA} / \mathrm{SCAl} 2005$ guideline update for percutaneous coronary intervention: a report of the American College of Cardiology/ American Heart Association Task Force on Practice guidelines. J Am Coll Cardiol. 2008;51(2):172-209.

14. Thygesen K, Alpert JS, White HD; Joint ESC/ACCF/AHA/ WHF Task Force for the Redefinition of Myocardial Infarction. Universal definition of myocardial infarction. Circulation. 2007;116(22):2634-53.

15. Cutlip DE, Windecker S, Mehran R, Boam A, Cohen DJ, van Es GA, Steg PG, Morel MA, Mauri L, Vranckx P, McFadden E, Lansky A, Hamon M, Krucoff MW, Serruys PW; Academy Research Consortium. Clinical end points in coronary stent trials. A case for standardized definitions. Circulation. 2007; 115(17):2344-51.

16. Antman EM, Cohen M, Bernink PJ, McCabe $\mathrm{CH}$, Horacek $\mathrm{T}$, Papuchis G, et al. The TIMI risk score for unstable angina/ non-ST elevation MI. A method for prognostication and therapeutic decision making. JAMA. 2000;284(7):835-42. Comment in: JAMA. 2000;284(7):876-8. 